\title{
Scaling relation for occulter manufacturing errors
}

Dan Sirbu1 ${ }^{1,3}$, Stuart B. Shaklan², N. Jeremy Kasdinn 1 , Robert J. Vanderbe

${ }^{1}$ Princeton University, ${ }^{2}$ NASA JPL, ${ }^{3}$ NASA ARC

\section{OBJECTIVE}

For directly imaging exoplanets, NASA is considering space mission designs that use an external occulter as the principal starlight suppression system. These occulter
designs range in diameter from 16 to $40 \mathrm{~m}$ and separation distance from 8,000 to $60,000 \mathrm{~km}$ for telescopes with primary diameters of 0.5 to $4 \mathrm{~m}(1-2)$.

Occulter shapes are solutions to an optimization problem (3) which seeks to maximize suppression in the shadow subject to constraints such as size, separation, and verified experimentally to demonstrate predicted on-orbit performance. Due to the large sizes and separations involved the experiment must be scaled to lab size (4-5).

We are currently expanding the existing experimental testbed at Princeton to enable scaling of occulters operating at flight Fresnel sizes. Here we examine the effect on

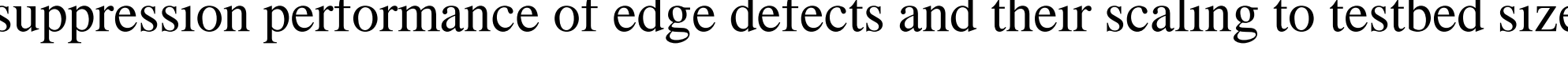

\section{OCCULTER SCALING}

For a plane wave incident on the space occulter, the electric field downstream at the telescope can be computed assuming an apodization function $\mathrm{A}(\mathrm{r})$ :

$$
E_{o}(\rho)=E_{0} e^{j k z}\left(1-\frac{k}{j z} \int_{0}^{R} e^{\frac{j k}{2 z}\left(r^{2}+\rho^{2}\right)} J_{0}\left(\frac{k r \rho}{z}\right) A(r) r d r\right)
$$

We can introduce scaling relationships that maintain the Fresnel numbers $r^{2} / \lambda z$ and $\rho^{2} / \lambda \mathrm{z}$ constant via a scaling factor a:

$$
E_{o}(\rho)=E_{0} e^{k j z^{\prime} a^{2}}\left(1-\frac{k}{j z^{\prime}} \int_{0}^{R^{\prime}} A\left(a r^{\prime}\right)\right) J_{0}\left(\frac{k r^{\prime} \rho^{\prime}}{z^{\prime}}\right) e^{\frac{k k^{\prime}}{2 z^{\prime}}\left(r^{\prime 2}+\rho^{\prime 2}\right)} r^{\prime} d r^{\prime}
$$

where in the above $\rho^{\prime} \rightarrow \rho / a, r^{\prime} \rightarrow r / a$, and $z^{\prime} \rightarrow z / a^{2}$

\section{MASK DESIGN}

We compare the design of the space occulter with other existing designs. We also design an ontes hing to minimize the diffraction effects from a finite-size:

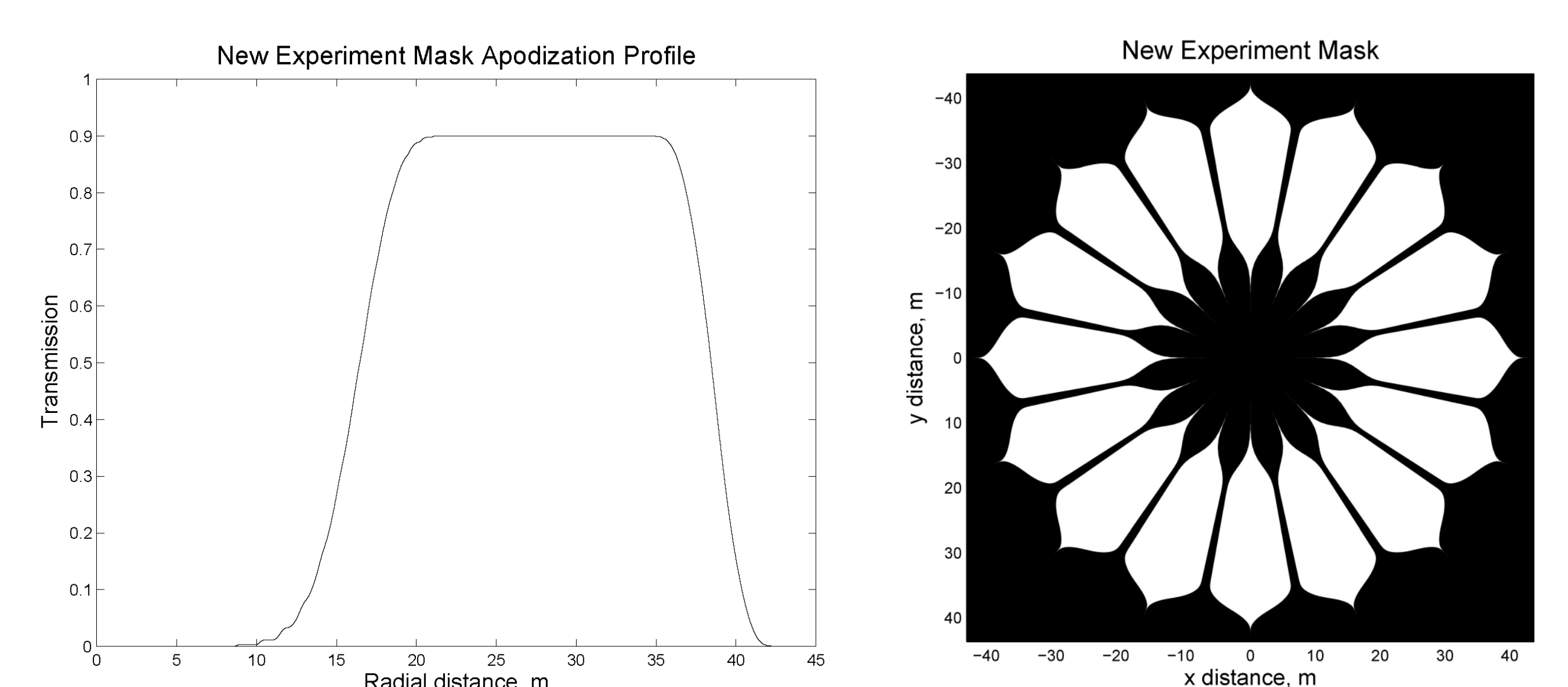

\begin{tabular}{lllll}
\hline Design & Occulter Radius, $R$ & Separation, $z$ & Wavelength, $\lambda$ & Fresnel Number \\
\hline THEIA & $20 \mathrm{~m}$ & $55,000 \mathrm{~km}$ & $600 \mathrm{~nm}$ & 12.1 \\
O3 & $17 \mathrm{~m}$ & $19,500 \mathrm{~km}$ & $600 \mathrm{~mm}$ & 24.7 \\
Current Experiment & $188 \mathrm{~m}$ & $97,000 \mathrm{~km}$ & $600 \mathrm{~nm}$ & 607.3 \\
Extended Experiment & $21.9 \mathrm{~m}$ & $55,000 \mathrm{~km}$ & $600 \mathrm{~nm}$ & 14.5 \\
\hline
\end{tabular}

Our testbed uses a diverging input beam to minimize optics aberrations. The occulter mask is placed at half-way of the total available distance to maximize its size.

\begin{tabular}{llllll}
\hline Parameter & Space Design & \multicolumn{2}{c}{ Collimated Scale } & \multicolumn{2}{c}{ Diverging Scale } \\
\hline Separation distance, $z$ & $55,000 \mathrm{~km}$ & $38 \mathrm{~m}$ & $50 \mathrm{~m}$ & $38 \mathrm{~m}$ & $50 \mathrm{~m}$ \\
Distance scale, $a$ & 1 & 1200 & 1050 & 1200 & 1050 \\
Source distance, $h$ & $\infty$ & $\infty$ & $\infty$ & $38 \mathrm{~m}$ & $50 \mathrm{~m}$ \\
Divergence escale, $\gamma$ & 1 & 1 & 1 & 1.4 & 1.4 \\
Inner radiusus, R $_{\text {inn }}$ & $21.9 \mathrm{~m}$ & $18.2 \mathrm{~mm}$ & $20.8 \mathrm{~mm}$ & $12.8 \mathrm{~mm}$ & $14.7 \mathrm{~mm}$ \\
Outer radius, $R_{\text {out }}$ & $43.7 \mathrm{~m}$ & $36.4 \mathrm{~mm}$ & $41.7 \mathrm{~mm}$ & $25.8 \mathrm{~mm}$ & $29.4 \mathrm{~mm}$ \\
Dark shadow radius, $\rho_{\text {dark }}$ & $2.6 \mathrm{~m}$ & $2.2 \mathrm{~mm}$ & $2.5 \mathrm{~mm}$ & $3.1 \mathrm{~mm}$ & $3.5 \mathrm{~mm}$ \\
Outer shadow radius, $\rho_{\text {out }}$ & $43.7 \mathrm{~m}$ & $36.4 \mathrm{~mm}$ & $41.7 \mathrm{~mm}$ & $51.5 \mathrm{~mm}$ & $57.8 \mathrm{~mm}$ \\
Telescope diameter, $D$ & $4 \mathrm{~m}$ & $3.3 \mathrm{~mm}$ & $3.8 \mathrm{~mm}$ & $3.3 \mathrm{~mm}$ & $3.8 \mathrm{~mm}$ \\
\hline
\end{tabular}

\section{OPTICAL PROPAGATIONS}

We use a 2D diffraction model first developed in (6) to perform a sensitivity analysis for determining the effect of laboratory-environment errors such as finite feature sizes and tunnel-induced diffraction effects.
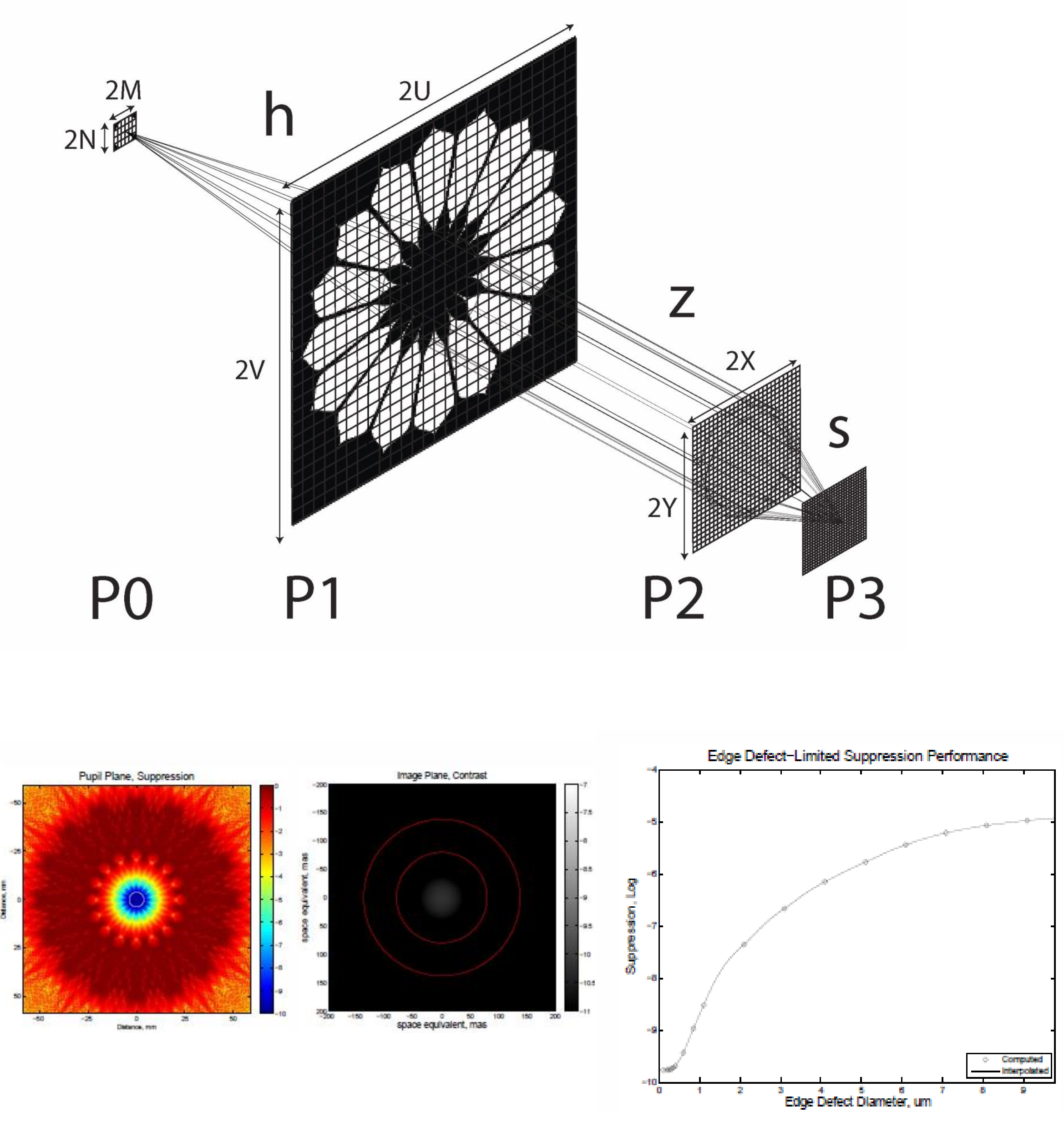

\section{○.}
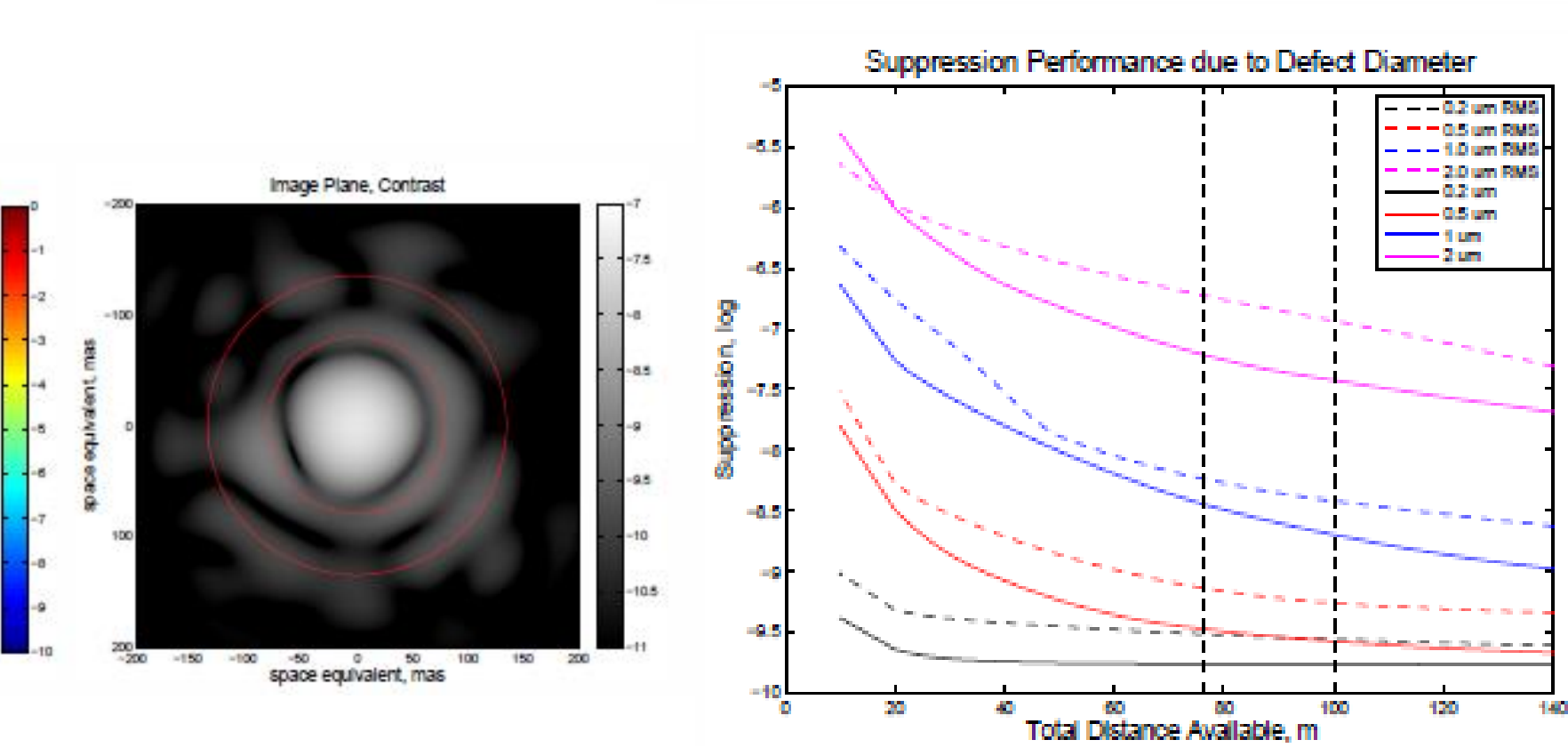

(Left) Sample propagations for increasing feature sizes (Right) Fitting of computed feature sizes for different propagation distances
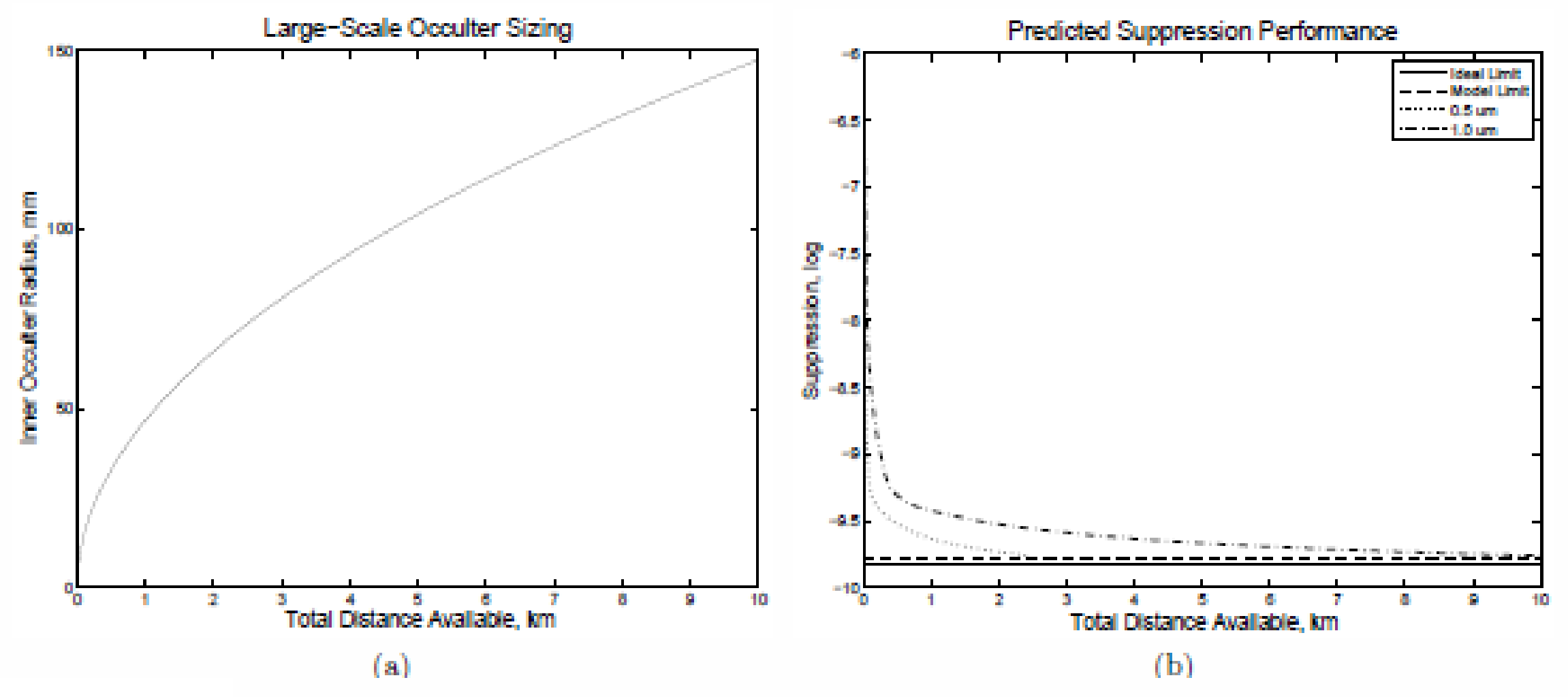

\section{ANALYTICAL MODEL}

The ratio of the pupil plane energy received from defects to the energy received from the point source is the suppression limit given for two cases depending on defect size
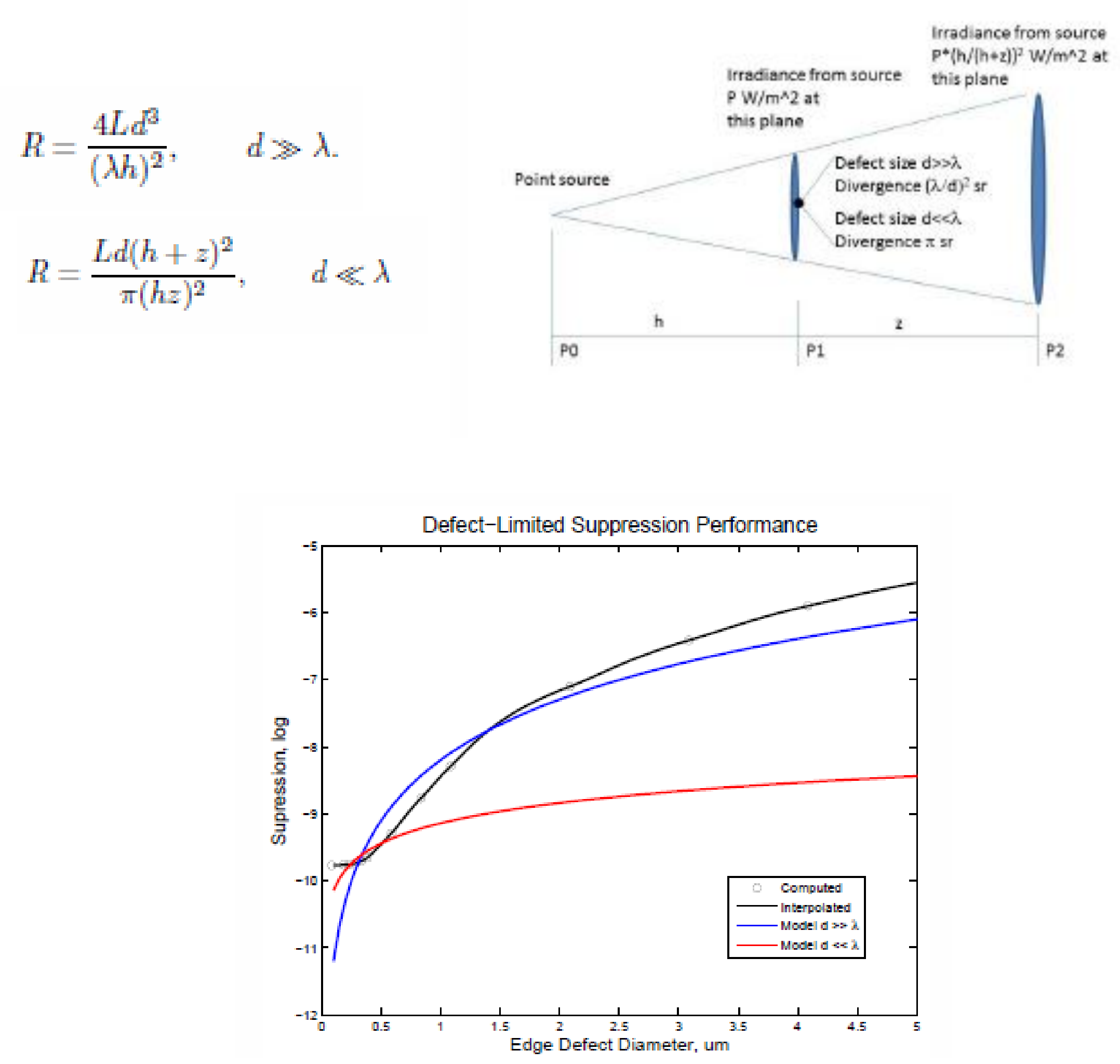

analytical model

We can thus model the expected effect on the performance of the occulter edge defects.

\section{CONCLUSIONS}

designed for realistic space mission parameters. This Ans has scaled to a laboratory size maintaining a constant Fresnel number. Optica propagations were performed hat verified the sappression and contrast performace

We have also developed an analytical model that can be used to

\section{REFERENCES}

(1) NJ Kasdin et al. A Telescope for Habitable Exoplanets and Interstellar/Intergalactic Astronomy. White Paper, 2009 .

NJ Kasdin et al. O3: The Occulting Ozone Observatory. AAS 2010 D Sirbu et al. "N

(6) D Sirbu et al "Diffractive analysis of limits of an occulter experiment," Proc. SPIE, Vol. 943,2014

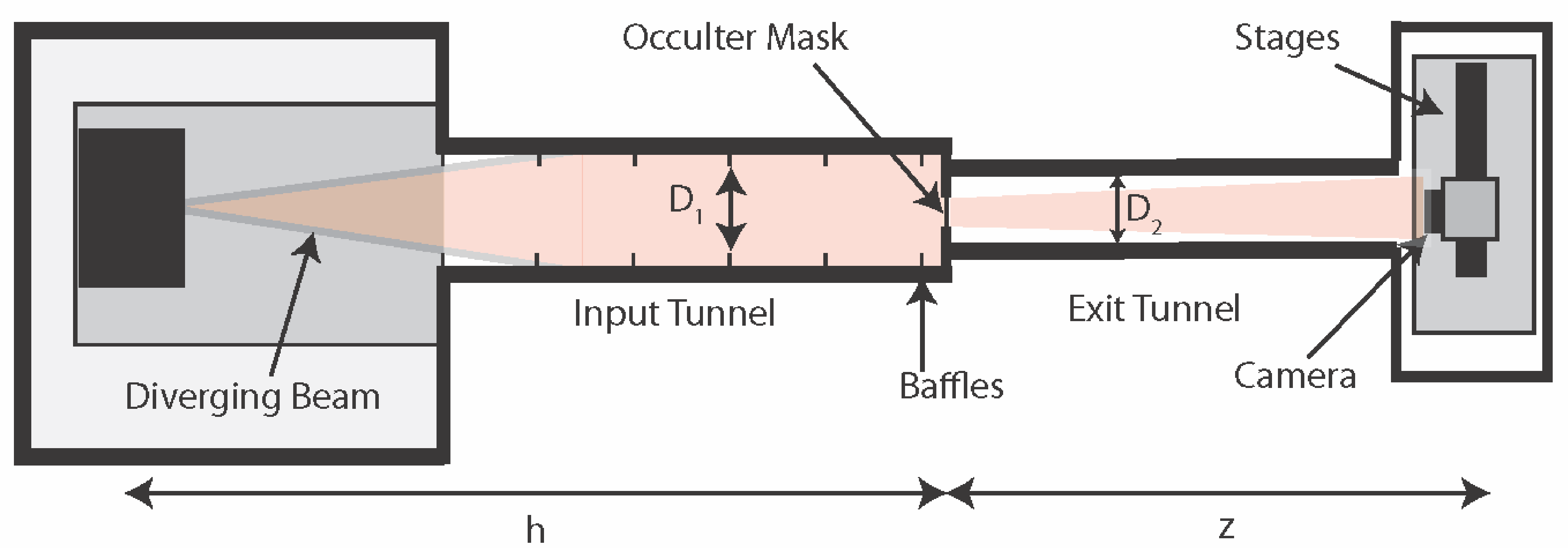

